\title{
A 2D threshold of the condylar-C1 interval to maximize identification of patients at high risk for atlantooccipital dislocation using computed tomography
}

\author{
Vijay M. Ravindra, MD, MSPH, ${ }^{1}$ Jay Riva-Cambrin, MD, MSc, ${ }^{3}$ Kevin P. Horn, MD, PhD, ${ }^{2}$ \\ Jason Ginos, MD, ${ }^{2}$ Russell Brockmeyer, BA, Jian Guan, MD, ${ }^{1}$ John Rampton, MD, ${ }^{2}$ and \\ Douglas L. Brockmeyer, MD'

\begin{abstract}
1Division of Pediatric Neurosurgery, Department of Neurosurgery, Primary Children's Hospital, University of Utah, and 2Department of Radiology, University of Utah School of Medicine, Salt Lake City, Utah; and 'Department of Clinical Neurosciences, Division of Pediatric Neurosurgery, University of Calgary, Alberta, Canada
\end{abstract}

\begin{abstract}
OBJECTIVE Measurement of the occipital condyle-C1 interval $(\mathrm{CCl})$ is important in the evaluation of atlantooccipital dislocation (AOD) in pediatric trauma patients. The authors studied a large cohort of children with and without AOD to identify a 2D measurement threshold that maximizes the diagnostic yield of the $\mathrm{CCl}$ on cervical spine $\mathrm{CT}$ scans obtained in trauma patients.
\end{abstract}

METHODS This retrospective, single-center study included all children who underwent CT of the cervical spine at Primary Children's Hospital from January 1, 2011, through December 31, 2014, for trauma evaluation. Bilateral CCl measurements in the coronal ( 3 measurements per side) and sagittal (4 measurements per side) planes were recorded. Using an iterative method, the authors determined optimal cutoffs for the maximal $\mathrm{CCl}$ in each plane in relation to AOD. The primary outcome was AOD requiring occipitocervical fusion.

RESULTS A total of 597 pediatric patients underwent cervical spine CT for trauma evaluation: 578 patients without AOD and 19 patients with AOD requiring occipitocervical fusion. The authors found a statistically significant correlation between $\mathrm{CCl}$ and age $(p<0.001)$, with younger patients having higher $\mathrm{CCls}$. Using a 2D threshold requiring a sagittal $\mathrm{CCl}$ $\geq 2.5 \mathrm{~mm}$ and a coronal $\mathrm{CCl} \geq 3.5 \mathrm{~mm}$ predicted AOD with a sensitivity of $95 \%$, a specificity of $73 \%$, positive predictive value of $10.3 \%$, and negative predictive value of $99 \%$. The accuracy of this $2 \mathrm{D}$ threshold was $84 \%$.

CONCLUSIONS In the present study population, age-dependent differences in the CCI were found on CT scans of the cervical spine in a large cohort of patients with and without AOD. A 2D CCI threshold as a screening method maximizes identification of patients at high risk for AOD while minimizing unnecessary imaging studies in children being evaluated for trauma.

https://thejns.org/doi/abs/10.3171/2016.10.PEDS16459

KEY WORDS occipital condyle-C1 interval; computed tomography; atlantooccipital dislocation; measurement; trauma; pediatrics; cervical spine

$\mathrm{T}$ RAUMATIC dislocation of the atlantooccipital joint, otherwise known as atlantooccipital dislocation (AOD), remains one of the most common fatal or disabling cervical spine injuries in children and adults. ${ }^{1-3}$, 5-7 Several studies have shown that AOD occurs twice as frequently in children as adults ${ }^{3}$ but that children also have better outcomes in spite of severe neurological deficits on presentation. ${ }^{10,11}$ Clearly, diagnostic schemes designed to improve the detection of AOD would enhance the management of this disorder by increasing diagnostic yield of trauma CT scans while ideally decreasing the overall number of unnecessary radiographic studies.

The occipital condyle- $\mathrm{C} 1$ interval $(\mathrm{CCI})^{9}$ has become an important measurement in defining traumatic injury at the craniocervical junction. The CCI directly measures the distance between the occipital condyle and the superior articular surface of $\mathrm{C}-1$ on CT scans. A greater distance represents injury to the occiput-C1 (Oc-C1) joint, with a value $\geq 4 \mathrm{~mm}$ chosen as the threshold for detection of AOD in most studies. ${ }^{?}$ 

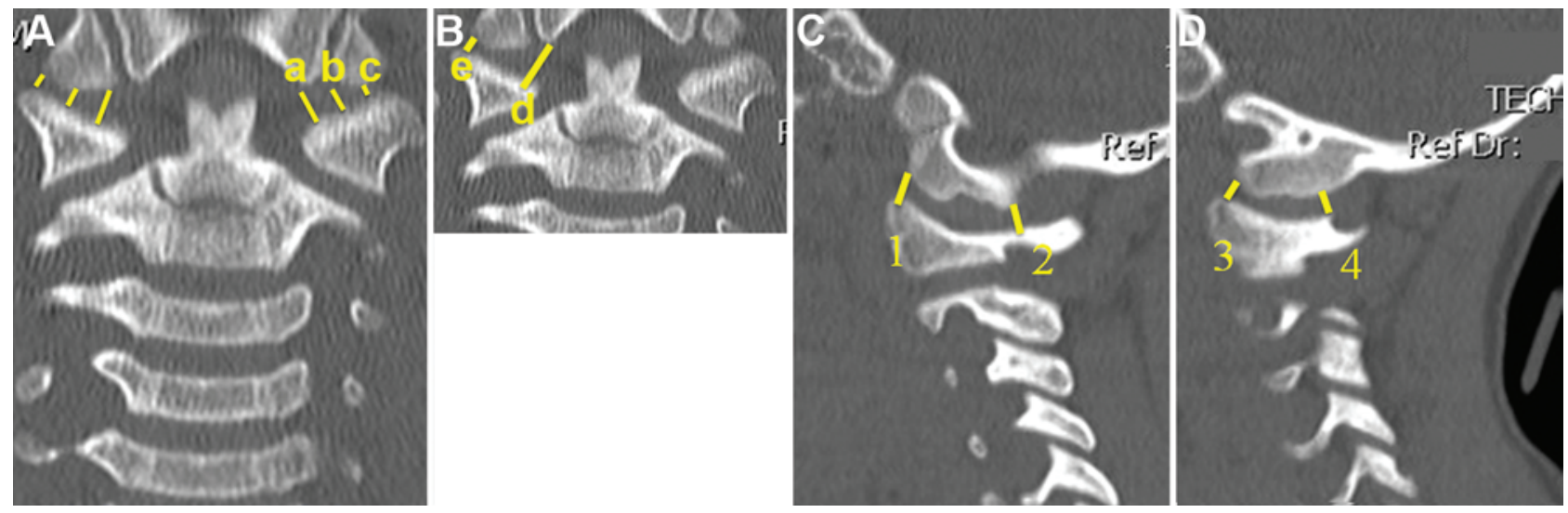

FIG. 1. A: Coronal reformatted CT image demonstrating the $\mathrm{CCl}$ measurements taken across the Oc-C1 joint: coronal medial measurement (a), coronal middle measurement (b), and coronal lateral measurement (c). B-D: Coronal (B) and sagittal (C and D) reformatted CT images demonstrating the $\mathrm{CCl}$ measurements taken across the $\mathrm{Oc}-\mathrm{C} 1$ joint: anterior portion of the joint (d) and posterior portion of the joint (e). 1 = medial anterior measurement; 2 = medial posterior measurement; $3=$ lateral anterior measurement; and 4 = lateral posterior measurement. Figure is available in color online only.

In 2007, Pang et al. ${ }^{9}$ defined the normal CCI distance in 89 children as $1.28 \pm 0.26 \mathrm{~mm}$ over a wide age range, from young children to teenagers; however, in practical use, the accuracy of the measurement has been called into question because of the presence of articular cartilage at the Oc-C1 joint (not seen on CT scans) and its effect on the CCI. The presence of normal articular cartilage at the $\mathrm{Oc}-\mathrm{Cl}$ joint artificially increases the $\mathrm{CCI}$ in young children, often past the 4-mm threshold for AOD, making it difficult to determine whether AOD is present and diminishing the value of the trauma cervical CT scan. Thus, establishing age-dependent CCI norms and improving the CCI threshold criteria for the diagnosis of AOD would benefit pediatric trauma patients. In fact, Vachhrajani et al..$^{13}$ recently concluded, based on the results from a series of 42 children, that age-dependent and -independent normal CT measurements of the upper cervical spine may help in further differentiating physiological and pathological states in children with traumatic injuries.

The goal of this study, by studying a large cohort of patients, was to establish normative parameters for the CCI in children that can be used to develop a 2D CCI imaging threshold for improving the diagnostic accuracy of AOD. An improvement in diagnostic accuracy could prevent any missed diagnoses of AOD while possibly minimizing the unnecessary use of radiographic imaging in pediatric trauma patients. The primary objective of this study was to identify specific radiographic measurements that characterize children at high risk for the presence of AOD. The secondary objective was to establish normal, age-based radiographic parameters of the pediatric cervical spine.

\section{Methods}

A single-center institutional review board-approved retrospective review of children was conducted to evaluate all patients in the Primary Children's Hospital trauma database treated from 2011 through 2014. We included all patients $\leq 18$ years of age who underwent a trauma evalua- tion involving a noncontrast CT scan of the cervical spine. All patients in the study cohort had successful clearance of their cervical spine. Patients in the comparison group with AOD were identified and analyzed separately.

The primary outcome was diagnosis of AOD requiring fusion surgery. All patients included in the comparison group satisfied one or more accepted radiographic criteria for AOD or presented with well-known clinical and MRI-documented features of AOD including neurological deficits localized to the cervicomedullary junction, tectorial membrane injury, posterior ligamentous injury, and $\mathrm{Oc}-\mathrm{C} 1$ capsular ligament injury.

\section{Radiographic Measurements}

The imaging sequences reviewed were part of a head and neck protocol used for trauma evaluation. In this protocol, nonoverlapping axial CT scans $(1.5-2.5 \mathrm{~mm})$ were obtained from the mid-clivus region to the bottom of C-2 . Corresponding sagittal and coronal reconstructions (2 $\mathrm{mm}$ ) were created to allow for a complete 3D visualization of the $2 \mathrm{Oc}-\mathrm{C} 1$ joints.

Radiographic information collected included CT measurements of the CCI in the coronal and sagittal planes. In the coronal plane, a total of 6 measurements were obtained, 3 on each side, representing the lateral, middle, and medial portions of the joint (Fig. 1A). A scout line was used to verify measurement along the midportion of the joint on a linked sagittal image. In the sagittal plane, a total of 8 measurements were obtained, 4 on each side, representing the lateral and medial edges of the joint with an anterior/posterior measurement for each (Fig. 1B). These metrics were chosen to obtain the most information possible about the joint space and clearly define the 3D joint anatomy. If MR images were obtained, the findings including the presence of $\mathrm{Oc}-\mathrm{C} 1$ joint injury, tectorial membrane disruption, and posterior ligamentous injury were recorded. Additional CT findings, including irregularities identified on flexion/extension CT imaging and the presence of dynamic instability, were also recorded. 
TABLE 1. Descriptive statistics and Pearson's correlation of measurements in patients without AOD in the coronal and sagittal planes

\begin{tabular}{cccccc}
\hline \multirow{2}{*}{ Variable } & \multicolumn{3}{c}{ Measurement in mm } & & \\
\cline { 2 - 3 } & Mean \pm SD & Median $(\mathrm{IQR})$ & Range & Pearson's Coefficient & p Value \\
\hline Coronal measurements & & & & $<$ \\
\hline Left coronal lateral & $1.61 \pm 0.59$ & $1.50(0.7)$ & $0.4-4.2$ & -0.27 & $<0.001$ \\
\hline Left coronal middle & $2.02 \pm 0.80$ & $1.90(1.1)$ & $0.6-5.4$ & -0.48 & $<0.001$ \\
\hline Left coronal medial & $2.65 \pm 1.15$ & $2.60(1.6)$ & $0.6-7.6$ & -0.43 & $<0.001$ \\
\hline Right coronal lateral & $1.63 \pm 0.64$ & $1.50(0.7)$ & $0.4-4.7$ & -0.25 & $<0.001$ \\
\hline Right coronal middle & $2.04 \pm 0.77$ & $2.0(1.1)$ & $0.6-4.7$ & -0.48 & $<0.001$ \\
\hline Right coronal medial & $2.70 \pm 1.10$ & $2.6(1.5)$ & $0.6-7.2$ & -0.47 & $<0.001$ \\
\hline Sagittal measurements & & & & -0.59 & $<0.001$ \\
\hline Left medial anterior & $2.0 \pm 0.83$ & $1.9(1.1)$ & $0.5-4.9$ & -0.48 & $<0.001$ \\
\hline Left medial posterior & $2.16 \pm 0.86$ & $2.0(1.2)$ & $0.7-5.5$ & -0.29 & $<0.001$ \\
\hline Left lateral anterior & $1.79 \pm 0.65$ & $1.7(0.9)$ & $0.6-4.8$ & -0.37 & $<0.001$ \\
\hline Left lateral posterior & $1.74 \pm 0.59$ & $1.7(0.8)$ & $0.6-4.5$ & -0.62 & $<0.001$ \\
\hline Right medial anterior & $2.03 \pm 0.79$ & $2.0(1.2)$ & $0.5-5.0$ & -0.51 & $<0.001$ \\
\hline Right medial posterior & $2.14 \pm 0.84$ & $2.1(1.1)$ & $0.6-5.6$ & -0.38 & $<0.001$ \\
\hline Right lateral anterior & $1.77 \pm 0.62$ & $1.7(0.8)$ & $0.4-4.3$ & -0.42 & $<0.001$ \\
\hline Right lateral posterior & $1.72 \pm 0.59$ & $1.6(0.7)$ & $0.5-4.6$ & &
\end{tabular}

\section{Statistical Analysis}

Data were summarized using means and standard deviations for continuous variables and counts and frequencies for categorical variables. Each of the 14 individual measurements (6 coronal, 8 sagittal) was compared with the outcome of AOD requiring fusion surgery by using t-tests. CCI values in both dimensions were compared with continuous age by using Pearson's correlation and with sex by using t-tests. Five individuals, including 3 radiologists and 2 neurosurgeons, measured the CCI values in an overlapping fashion to assess for agreement among observers; the interrater reliability was calculated using cutoffs for the CCI (Cohen's kappa statistic) and by treating the CCI as a continuous variable (Pearson's and Spearman's correlation coefficient).

We then used similar analyses (t-tests) to compare maximal sagittal and maximal coronal measurements for each patient with whether the patient had AOD requiring fusion surgery. We used an iterative method of assessing measurements to identify cutoffs for maximizing sensitivity, specificity, positive predictive value, and negative predictive value for both coronal and sagittal measurements. We then combined the 2 cutoffs for both the coronal and sagittal planes and reanalyzed the data set to determine the sensitivity, specificity, positive predictive value, and negative predictive value for this $2 \mathrm{D}$ prediction rule. Statistical significance was established using a cutoff of $p<$ 0.05 . Data were analyzed using SAS version 9.3 software.

\section{Results}

A total of 597 patients underwent cervical spine CT for trauma evaluation: 578 patients without AOD and 19 patients with AOD requiring OC fusion. Of the 578 pa- tients without AOD injury, 365 (63.2\%) were male. The mean age of the patients without AOD was $8.7 \pm 5.1$ years (range 3 weeks-18 years, median 9 years). The mean and standard deviation, median and interquartile range, and range for each measurement are listed in Table 1. Pearson's correlation testing demonstrated an inverse relationship (smaller measurement with increasing age) as well as a statistically significant correlation coefficient between each measurement as a function of age (Table 1). The maximal measurement for both coronal (Fig. 2A) and sagittal (Fig. 2B) CCI as a function of age was plotted. The difference in the mean measurement for each measurement for females and males was also statistically significant for all coronal measurements and in all but 2 of the sagittal measurements (sagittal right lateral anterior, $p=0.83$; and sagittal right lateral posterior, $\mathrm{p}=0.09$ ) (Table 2). The Cohen's kappa statistic for interobserver reliability was 0.66 in the coronal plane and 0.50 in the sagittal plane, and as a continuous variable, the Pearson's correlation coefficient in the coronal plane was 0.82 and in the sagittal plane was 0.85 , whereas the Spearman's correlation coefficient in the coronal plane was 0.80 and in the sagittal plane was 0.85 $(\mathrm{p}<0.001)$.

The mean age of the 19 patients with AOD was $5.7 \pm$ 3.5 years, and their younger age was statistically significant compared with the age of the children without AOD $(\mathrm{p}=0.01)$. There was no significant difference in sex between the groups $(\mathrm{p}=0.47)$, with males representing $73.7 \%$ (14 patients) of the AOD group and $63.2 \%$ of the non-AOD group. When comparing patients without AOD and those with AOD, there were statistically significant differences in whether an MR image of the cervical spine was obtained $(\mathrm{p}<0.001)$, in the rates of $\mathrm{Oc}-\mathrm{C} 1$ capsular 

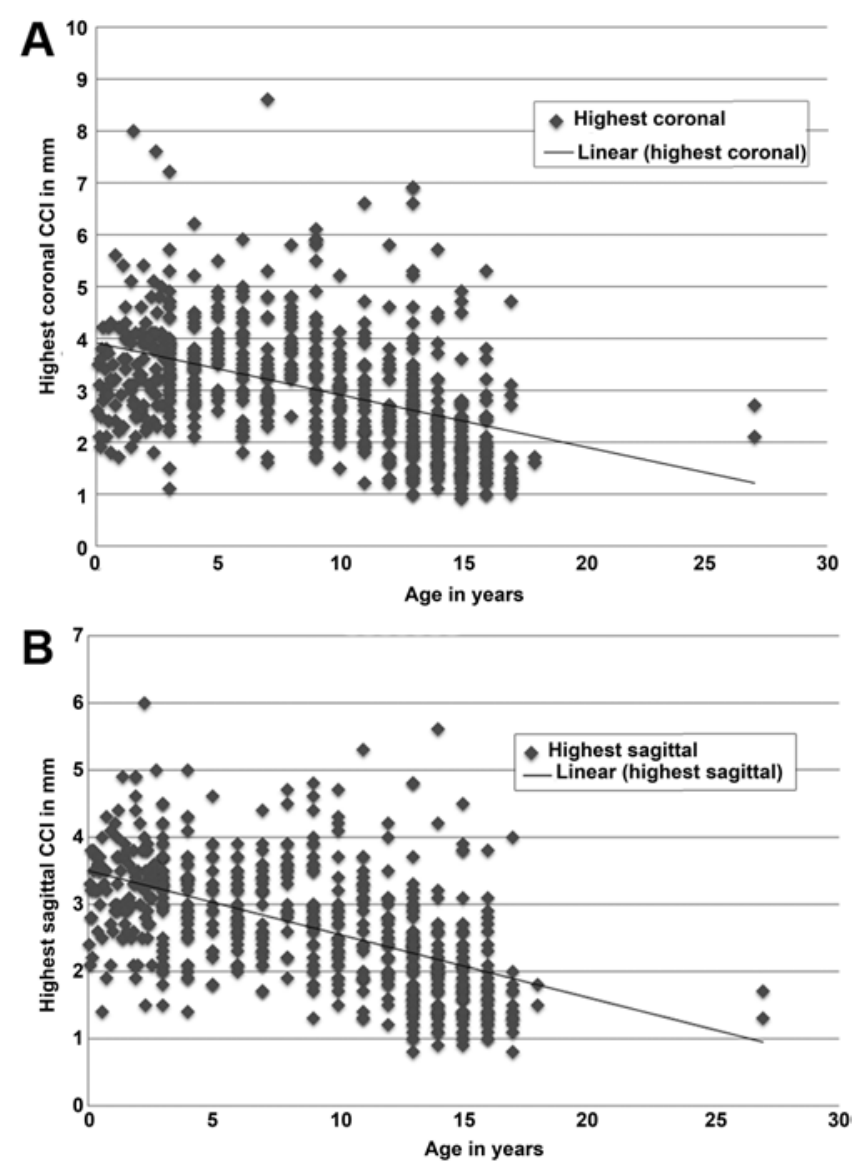

FIG. 2. A: The highest coronal $\mathrm{CCl}(\mathrm{mm})$ for each patient plotted as a function of age (years). B: The highest sagittal $\mathrm{CCl}(\mathrm{mm})$ for each patient plotted as a function of age (years).

injury $(0.4 \%$ vs $73.7 \%, \mathrm{p}<0.001)$ and tectorial membrane disruption $(0.4 \%$ vs $36.8 \%, \mathrm{p}<0.001)$, and in the posterior ligamentous injury findings identified on MRI (2.9\% vs $79 \%$, respectively; $\mathrm{p}<0.001)$. Only a few flexion/extension CT studies were performed in either group (6 total), but subluxation was significantly more frequent on flexion/extension CT scans in the AOD group ( $0.2 \%$ vs $5.3 \%$, $\mathrm{p}<0.001$ ), as would be expected (Supplemental Table 1). Table 3 demonstrates the mean measurement characteristics for each parameter in patients without AOD and patients with AOD requiring fusion; there were statistically significant differences between all measurements in the 2 groups of patients.

In the face of multiple measurements, we decided to use the maximal value for both coronal and sagittal measurements, which serves to simplify measurement and ensures ease of clinical use. An iterative process was used to determine optimal cutoffs for both the maximal sagittal CCI and the maximal coronal $\mathrm{CCI}$, with the driving principle to prioritize sensitivity (minimize the chance of missing an AOD) and secondarily achieve the highest specificity possible (minimize the false positives, which require further testing and expense). Using only the maximal CCI in the sagittal plane with a cutoff of $\geq 2.5 \mathrm{~mm}$ as a predictor of AOD requiring fusion yielded a sensitivity of $100 \%$,
TABLE 2. Comparison of mean measurements by sex in the coronal and sagittal plane in the patients without AOD

\begin{tabular}{cccc}
\hline & \multicolumn{2}{c}{ Measurement in mm } & $p$ \\
\cline { 2 - 3 } Measurement Type & Female & Male & Value \\
\hline Mean coronal measurements & & & \\
\hline Left coronal lateral & 1.5 & 1.7 & $<0.001$ \\
\hline Left coronal middle & 1.8 & 2.1 & $<0.001$ \\
\hline Left coronal medial & 2.4 & 2.8 & $<0.001$ \\
\hline Right coronal lateral & 1.4 & 1.7 & $<0.001$ \\
\hline Right coronal middle & 1.9 & 2.1 & $<0.001$ \\
\hline Right coronal medial & 2.5 & 2.8 & 0.002 \\
\hline Mean sagittal measurements & & & \\
\hline Left medial anterior & 1.8 & 2.1 & $<0.001$ \\
\hline Left medial posterior & 2.1 & 2.2 & 0.05 \\
\hline Left lateral anterior & 1.7 & 1.9 & $<0.001$ \\
\hline Left lateral posterior & 1.7 & 1.8 & 0.01 \\
\hline Right medial anterior & 1.9 & 2.1 & 0.004 \\
\hline Right medial posterior & 2.1 & 2.1 & 0.83 \\
\hline Right lateral anterior & 1.6 & 1.8 & $<0.001$ \\
\hline Right lateral posterior & 1.7 & 1.8 & 0.09 \\
\hline
\end{tabular}

specificity of $44 \%$, percentage of misclassification of $54 \%$, positive predictive value of $5.5 \%$, and negative predictive value of $100 \%$. Using only the maximal CCI in the coronal plane with a cutoff $\geq 3.5 \mathrm{~mm}$ as a predictor of AOD requiring fusion yielded a sensitivity of $100 \%$, specificity of $52 \%$, positive predictive value of $6.5 \%$, and negative predictive value of $100 \%$. Combining the 2 cutoffs by using a $2 \mathrm{D}$ threshold requiring a sagittal $\mathrm{CCI} \geq 2.5 \mathrm{~mm}$ and a coronal $\mathrm{CCI} \geq 3.5 \mathrm{~mm}$ yielded a sensitivity of $95 \%$, an improved specificity of $73 \%$, positive predictive value of $10.3 \%$, negative predictive value of $99 \%$, and percentage of misclassification of $26.2 \%$. The accuracy of this 2D threshold was $84 \%$ (Table 4).

\section{Discussion}

\section{Screening for AOD}

We have successfully identified a 2D CCI threshold that maximizes identification of children at high risk for AOD; this threshold uses a sagittal measurement of 2.5 $\mathrm{mm}$ and a coronal measurement of $3.5 \mathrm{~mm}$ as cutoffs. Furthermore, we found that younger children had significantly higher CCI values than older children, and age-specific norms can be approximated from our data.

Previous work has shown that AOD is a challenging diagnosis to make in the pediatric trauma population. A variety of diagnostic criteria have been proposed over the years, but the 2007 paper by Pang et al. ${ }^{9}$ demonstrated the importance of the CCI based on a 3-part hypothesis: 1) the $\mathrm{Oc}-\mathrm{C} 1$ joint is held tightly together by strong ligaments as part of a narrow complex (the CCI); 2) there is a high level of symmetry between the left and right $\mathrm{Oc}-\mathrm{C} 1$ joints; and 3 ) forces that disrupt the $\mathrm{Oc}-\mathrm{Cl}$ joint will enlarge the $\mathrm{CCI}$ and/or produce left-right asymmetry in the joint. Pang et al. chose a CCI of $4.0 \mathrm{~mm}$, or $10 \mathrm{SDs}$ above the mean, as 
TABLE 3. Measurement characteristics in children with no injury and those with $A O D$ requiring fusion

\begin{tabular}{|c|c|c|c|}
\hline \multirow[b]{2}{*}{ Measurement Type } & \multicolumn{2}{|c|}{ Measurement in $\mathrm{mm}^{*}$} & \multirow[b]{2}{*}{$\begin{array}{c}p \\
\text { Value }\end{array}$} \\
\hline & $\begin{array}{l}\text { No Injury } \\
(n=578)\end{array}$ & $\begin{array}{c}\text { AOD } \\
(n=19)\end{array}$ & \\
\hline \multicolumn{4}{|l|}{ Coronal measurements } \\
\hline Left coronal lateral & $1.6 \pm 0.6$ & $2.1 \pm 0.7$ & $<0.001$ \\
\hline Left coronal middle & $2.0 \pm 0.8$ & $2.8 \pm 0.8$ & $<0.001$ \\
\hline Left coronal medial & $2.7 \pm 1.2$ & $3.6 \pm 0.8$ & $<0.001$ \\
\hline Right coronal lateral & $1.6 \pm 0.6$ & $2.8 \pm 1.5$ & $<0.001$ \\
\hline Right coronal middle & $2.0 \pm 0.8$ & $3.8 \pm 1.3$ & $<0.001$ \\
\hline Right coronal medial & $2.7 \pm 1.1$ & $4.5 \pm 1.1$ & $<0.001$ \\
\hline Maximal coronal CCI & $3.0 \pm 1.2$ & $4.6 \pm 1.3$ & $<0.001$ \\
\hline \multicolumn{4}{|l|}{ Sagittal measurements } \\
\hline Left medial anterior & $2.0 \pm 0.8$ & $2.8 \pm 0.8$ & $<0.001$ \\
\hline Left medial posterior & $2.2 \pm 0.9$ & $3.3 \pm 0.9$ & $<0.001$ \\
\hline Left lateral anterior & $1.8 \pm 0.6$ & $2.3 \pm 0.8$ & $<0.001$ \\
\hline Left lateral posterior & $1.7 \pm 0.6$ & $3.0 \pm 0.9$ & $<0.001$ \\
\hline Right medial anterior & $2.0 \pm 0.8$ & $3.2 \pm 1.1$ & $<0.001$ \\
\hline Right medial posterior & $2.1 \pm 0.8$ & $3.4 \pm 1.1$ & $<0.001$ \\
\hline Right lateral anterior & $1.8 \pm 0.6$ & $2.8 \pm 1.0$ & $<0.001$ \\
\hline Right lateral posterior & $1.7 \pm 0.6$ & $3.2 \pm 1.2$ & $<0.001$ \\
\hline Maximal sagittal $\mathrm{CCl}$ & $2.7 \pm 0.9$ & $4.0 \pm 1.0$ & $<0.001$ \\
\hline
\end{tabular}

${ }^{*}$ Measurements are presented as the mean \pm SD.

the cutoff value for diagnosing AOD. These principles are also relevant in adults. Recently, Martinez-del-Campo et al. ${ }^{8}$ found a CCI cutoff of $1.5 \mathrm{~mm}$ using a similar method of comparison in a cohort of 81 adult patients -59 without AOD and 22 with AOD.

In practical use, however, we have found that the CCI screening criteria proposed by Pang et al. ${ }^{9}$ were, at times, missing significantly unstable atlantooccipital injuries that required a subsequent fusion. Thus, the goal of our study was to increase the sensitivity and accuracy of CCI screening by establishing normal CCI values from a large cohort of patients and using those values to create a novel 2D threshold criterion for the diagnosis of AOD. There was a statistically significant difference between the mean characteristics for each measurement in the 2 groups of patients (Table 3). We attempted to simplify the information for clinical application by using the maximal CCI for each patient in the sagittal and coronal plane and using cutoffs to calculate sensitivity, specificity, positive and negative predictive value, and percentage of misclassification (Table 4). In doing so, we provide practitioners with an easy-to-use bedside tool with which to evaluate pediatric trauma patients. Using the 2D threshold technique, only $1(5.3 \%)$ of 19 AOD patients in our cohort of 597 would be missed by radiographic criteria alone whereas applying the criteria defined by Pang et al. ( $\geq 4 \mathrm{~mm}$ in either direction) would have missed 3 (15.8\%) of 19 (Table 4). Given the overall severity of AOD and its implications for lifelong morbidity, any predictive threshold should maximize sensitivity while secondarily balancing specificity. We contend that a $15.8 \%$ AOD miss rate is not acceptable,
TABLE 4. A $2 \times 2$ table for screening of AOD using the data set of 597 patients

\begin{tabular}{lccc}
\hline \multicolumn{1}{c}{ Method } & AOD Requiring Fusion & No Injury & Total \\
\hline 2D method* & & & \\
\hline $\begin{array}{c}\text { Coronal } \mathrm{CCI} \geq 3.5 \mathrm{~mm}, \\
\text { sagittal } \mathrm{CCI} \geq 2.5 \mathrm{~mm}\end{array}$ & 18 & 156 & 174 \\
\hline All others & 1 & 422 & 423 \\
\hline Total & 19 & 578 & 597 \\
\hline $\begin{array}{l}\text { Pang's criteria for AOD } \dagger \\
\text { Any sagittal or coronal } \\
\text { measurement } \geq 4 \mathrm{~mm}\end{array}$ & 16 & 124 & 140 \\
\hline All others & 3 & 454 & 457 \\
\hline Total & 19 & 578 & 597 \\
\hline
\end{tabular}

* Sensitivity $95 \%$; specificity $73 \%$; positive predictive value $10.3 \%$; negative predictive value $99 \%$; percentage of misclassification $26.2 \%$; and model accuracy $84 \%$.

† Sensitivity $84 \%$; specificity $78.5 \%$; positive predictive value $11.4 \%$; negative predictive value $99 \%$; and percentage of misclassification $21.3 \%$.

especially while saving only 32 extra images in false positives (improved specificity from $73 \%$ using our criteria to $78 \%$ using the criteria of Pang et al.).

The accuracy of this 2D threshold was $84 \%$, indicating a good fit for the model. It is desirable to have a highly sensitive test as a screening tool to rule out pathology. In addition, the high negative predictive value also adds to the practicality of using a $2 \mathrm{D}$ method in detecting AOD. Interrater reliability between neurosurgeons and radiologists yielded a Cohen's kappa statistic of 0.66 in the coronal plane (good agreement) and 0.50 in the sagittal plane (moderate agreement). The high Pearson's and Spearman's correlations using continuous data in both planes $(p<0.001)$ indicate a high level of correlation between different observers. Thus, the measurement technique and parameters can be used by practitioners in all specialties.

This is the first report of using a 2D threshold for measuring CCI and cutoff values determined in a large healthy population and one of patients with AOD. We postulate that application of this algorithm will significantly improve the diagnosis of AOD in the pediatric population.

\section{Age-Dependent Changes in $\mathrm{CCl}$}

In contrast to the findings of Pang et al., we identified a significant association between age and CCI for each sagittal and coronal measurement (Table 1); the trend line further enhances this relationship between age and maximal CCI in the coronal and sagittal planes (Fig. 2). There appears to be significant tightening of the joint space as patients reach 12 years of age and older. This finding is supported by a report from Smith et al., ${ }^{12}$ who demonstrated significant variability in the CCI across age groups in a cohort of 124 patients. Age-related changes in the CCI have been reported previously., ${ }^{4,13}$ Of particular interest in our study are the CCI measurements of patients 5 years of age or younger. In that age group, the mean CCI was $\geq 3 \mathrm{~mm}$ in the sagittal plane and $\geq 3.5 \mathrm{~mm}$ in the coronal plane, making it possible to suggest that specific age- 
dependent CCI cutoffs could be identified; however, this was beyond the scope of our study. Our findings also show there is a significant difference between sexes (Table 2) in the CCI, which may implicate the need for age- and sexspecific norms to be used in the future.

\section{MRI and Flexion/Extension CT Imaging}

All 19 patients diagnosed with AOD requiring fusion had MRI studies available for review. In all 19 patients there was evidence of craniovertebral junction disruption demonstrated by hyperintensity in the occipitoatlantal joint capsule or tectorial membrane injury. The comparison of uninjured patients with AOD patients revealed significant differences not only in each CCI measurement but also in MRI findings (Oc-C1 capsular injury, tectorial membrane injury, posterior ligamentous injury), as well as the utilization of flexion/extension CT imaging (Supplemental Table 1). These findings are not surprising given the primary outcome of patients diagnosed with AOD but do support the underlying comparison between injured and uninjured states.

\section{Limitations}

There were a limited number of patients previously diagnosed with AOD requiring fusion in our patient population, making it difficult to draw definitive conclusions regarding risk factors. Because this was a single-center retrospective cohort, the information gathered is limited by the accuracy and availability of the medical record and imaging and a potentially homogeneous patient population. Additionally, the applicability of the findings may be limited in situations where access to CT scanning is challenging. Although we believe the 2D method for detecting AOD is accurate, clinical evaluation and decision making should be paramount when making all diagnostic and treatment decisions. Validation of these parameters as a screening tool is necessary prior to widespread implementation.

\section{Conclusions}

Using a large cohort of children without AOD, as well as patients with AOD, we have successfully identified a 2D CCI threshold that maximizes identification of patients at high risk for AOD. As a screening tool, this will help in maximizing the diagnosis and management of AOD in the pediatric trauma population while minimizing unnecessary imaging studies in children. Further studies for age-dependent and sex-specific thresholds for the CCI are necessary.

\section{Acknowledgments}

We thank Richard Holubkov, $\mathrm{PhD}$, for his assistance with the statistical analysis and Kristin Kraus, MSc, for editorial assistance in preparing this paper.

\section{References}

1. Adams VI: Neck injuries: I. Occipitoatlantal dislocation-a pathologic study of twelve traffic fatalities. J Forensic Sci 37:556-564, 1992
2. Ahuja A, Glasauer FE, Alker GJ Jr, Klein DM: Radiology in survivors of traumatic atlanto-occipital dislocation. Surg Neurol 41:112-118, 1994

3. Alker GJ Jr, Oh YS, Leslie EV: High cervical spine and craniocervical junction injuries in fatal traffic accidents: a radiological study. Orthop Clin North Am 9:1003-1010, 1978

4. Bertozzi JC, Rojas CA, Martinez CR: Evaluation of the pediatric craniocervical junction on MDCT. AJR Am J Roentgenol 192:26-31, 2009

5. Bucholz RW, Burkhead WZ: The pathological anatomy of fatal atlanto-occipital dislocations. J Bone Joint Surg Am 61:248-250, 1979

6. Lee C, Woodring JH, Goldstein SJ, Daniel TL, Young AB, Tibbs PA: Evaluation of traumatic atlantooccipital dislocations. AJNR Am J Neuroradiol 8:19-26, 1987

7. Lee C, Woodring JH, Walsh JW: Carotid and vertebral artery injury in survivors of atlanto-occipital dislocation: case reports and literature review. J Trauma 31:401-407, 1991

8. Martinez-Del-Campo E, Kalb S, Soriano-Baron H, Turner JD, Neal MT, Uschold T, et al: Computed tomography parameters for atlantooccipital dislocation in adult patients: the occipital condyle-C1 interval. J Neurosurg Spine 24:535545, 2016

9. Pang D, Nemzek WR, Zovickian J: Atlanto-occipital dislocation: part $1-$ normal occipital condyle-C1 interval in 89 children. Neurosurgery 61:514-521, 2007

10. Pang D, Sun P: Pediatric vertebral column and spinal cord injuries, in Winn HR (ed): Youmans Neurological Surgery. Philadelphia: Saunders, 2004

11. Pang D, Wilberger JE Jr: Traumatic atlanto-occipital dislocation with survival: case report and review. Neurosurgery 7:503-508, 1980

12. Smith P, Linscott LL, Vadivelu S, Zhang B, Leach JL: Normal development and measurements of the occipital condyleC1 interval in children and young adults. AJNR Am J Neuroradiol 37:952-957, 2016

13. Vachhrajani S, Sen AN, Satyan K, Kulkarni AV, Birchansky SB, Jea A: Estimation of normal computed tomography measurements for the upper cervical spine in the pediatric age group. J Neurosurg Pediatr 14:425-433, 2014

\section{Disclosures}

The authors report no conflict of interest concerning the materials or methods used in this study or the findings specified in this paper.

\section{Author Contributions}

Conception and design: DL Brockmeyer, Ravindra, Riva-Cambrin. Acquisition of data: all authors. Analysis and interpretation of data: Ravindra, Riva-Cambrin. Drafting the article: all authors. Critically revising the article: all authors. Reviewed submitted version of manuscript: all authors. Approved the final version of the manuscript on behalf of all authors: DL Brockmeyer.

\section{Supplemental Information \\ Online-Only Content}

Supplemental material is available with the online version of the article.

Supplemental Table 1. https://thejns.org/doi/suppl/10.3171/ 2016.10.PEDS16459.

\section{Correspondence}

Douglas L. Brockmeyer, Division of Pediatric Neurosurgery, Department of Neurosurgery, Primary Children's Hospital, $100 \mathrm{~N}$ Mario Capecchi Dr., Salt Lake City, UT 84113. email: neuropub@ hsc.utah.edu. 